\title{
CENTIMETER-LEVEL, ROBUST GNSS-AIDED INERTIAL POST-PROCESSING FOR MOBILE MAPPING WITHOUT LOCAL REFERENCE STATIONS
}

\author{
J. J. Hutton ${ }^{\text {a }}$, N. Gopaul ${ }^{\text {a }}$, X. Zhang ${ }^{\text {a }}$, J. Wang ${ }^{\text {a }}$, V. Menon ${ }^{\text {a }}$, D. Rieck ${ }^{b}$, A. Kipka ${ }^{\text {, F. Pastor }}{ }^{\text {b }}$ \\ a Trimble Navigation Limited, 85 Leek Cr., Richmond Hill, Ontario, Canada L4B 3B3 - (jhutton, ngopaul, xzhang, jhwang, \\ vmenon)@applanix.com \\ b Trimble Navigation Limited, Haringstrasse 19, Hohenkirchen-Siegertsbrunn Munich, 85635, Germany - (Daniel_Rieck, \\ Adrian_Kipka, Fabian_Pastor)@trimble.com
}

\section{ICWG III/I}

KEY WORDS: Differential GNSS, Sensor Orientation, Mobile Mapping, GNSS-Aided Inertial, Georeferenicng, Airborne Mapping, Direct Geroeferencing, PPP

\begin{abstract}
:
For almost two decades mobile mapping systems have done their georeferencing using Global Navigation Satellite Systems (GNSS) to measure position and inertial sensors to measure orientation. In order to achieve cm level position accuracy, a technique referred to as post-processed carrier phase differential GNSS (DGNSS) is used. For this technique to be effective the maximum distance to a single Reference Station should be no more than $20 \mathrm{~km}$, and when using a network of Reference Stations the distance to the nearest station should no more than about $70 \mathrm{~km}$. This need to set up local Reference Stations limits productivity and increases costs, especially when mapping large areas or long linear features such as roads or pipelines.
\end{abstract}

An alternative technique to DGNSS for high-accuracy positioning from GNSS is the so-called Precise Point Positioning or PPP method. In this case instead of differencing the rover observables with the Reference Station observables to cancel out common errors, an advanced model for every aspect of the GNSS error chain is developed and parameterized to within an accuracy of a few $\mathrm{cm}$. The Trimble Centerpoint RTX positioning solution combines the methodology of PPP with advanced ambiguity resolution technology to produce $\mathrm{cm}$ level accuracies without the need for local reference stations. It achieves this through a global deployment of highly redundant monitoring stations that are connected through the internet and are used to determine the precise satellite data with maximum accuracy, robustness, continuity and reliability, along with advance algorithms and receiver and antenna calibrations.

This paper presents a new post-processed realization of the Trimble Centerpoint RTX technology integrated into the Applanix POSPac MMS GNSS-Aided Inertial software for mobile mapping. Real-world results from over 100 airborne flights evaluated against a DGNSS network reference are presented which show that the post-processed Centerpoint RTX solution agrees with the DGNSS solution to better than $2.9 \mathrm{~cm}$ RMSE Horizontal and $5.5 \mathrm{~cm}$ RMSE Vertical. Such accuracies are sufficient to meet the requirements for a majority of airborne mapping applications.

\section{INTRODUCTION}

\subsection{Mobile Mapping}

As its name implies, mobile mapping involves the collection of data to make map products while in continuous motion. While one can argue that making soundings using a lead line from a slow moving ship qualifies as mobile mapping, it was not until the advent of aerial photogrammetry that the term really became applicable. Of course today mobile mapping is done from virtually any moving platform with a plethora of different digital sensors, ranging from sophisticated Geiger-mode LiDARs to the low-cost cameras found in smart phones.

The common theme to mobile mapping is collecting geospatial information as quickly, accurately and cost-effectively as possible, to support a number of different applications such as disaster recovery, autonomous vehicle navigation, GIS, asset monitoring, flood plain mapping and more.

\subsection{GNSS-Aided Inertial Processing for Mobile Mapping}

The use of GNSS and inertial to improve the efficiency of making maps from mobile platforms through Direct Georeferencing was pioneered in the mid 1990's on traditional airborne film cameras (Hutton et al, 2005), and has now become a standard method of georeferencing data collected from the land, sea and air.

The basic concept of Direct Georeferencing is to use highly accurate measurements of geographic position and orientation to geo-code each pixel or point collected by a calibrated imaging sensor, thus enabling map data products to be created without the use of extensive ground information (Mostafa et al, 2001).

The position and orientation measurements are generated using GNSS-aided Inertial processing in real-time and postprocessing. In this architecture measurements from the GNSS are used to control the errors of the navigation solution computed by integrating acceleration and angular rate measurements made by an Inertial Measurement Unit (IMU) into position, velocity and orientation (Figure 1). In such an 
architecture the absolute accuracy of the position solution is controlled by the accuracy of the GNSS measurements, while the accuracy of the orientation measurements is a function of the inertial sensors and vehicle dynamics.

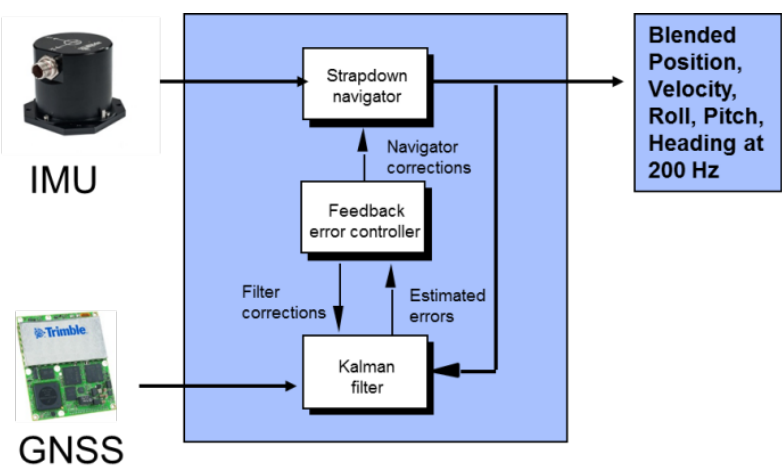

Figure 1: GNSS-Aided Inertial Architecture

In most cases the solution used to generate the final map product is generated post-mission, which has several key advantages:

- Higher accuracy can be achieve through forward and reverse-time processing

- Anomalies in data can be detected and repaired

- More advanced, spatially based and computationally intensive algorithms can be employed

- Complex and costly real-time data links are not required

- Regional or global GNSS augmentation services can be accessed via the internet

In order to achieve the cm-level accurate position most mapping products require, it is necessary to augment the basic GNSS system in order to correct it's measurements for systematic errors, which include receiver errors, satellite errors (clock, orbit) and propagation errors (ionospheric and tropospheric delays). The most accurate GNSS augmentation methodologies include carrier phase differential GNSS (DGNSS) and Precise Point Positioning (PPP).

\subsection{Carrier Phase Differential GNSS}

Differential GNSS (DGNSS) involves the simultaneous observation of the satellite radio signals at the rover receiver and at one or more reference receivers, and then differencing the signals to cancel out common errors. Carrier Phase Differential GNSS is a method of DGNSS that uses the carrier signals (L1, L2, L5) to make more accurate range measurements to the satellites (carrier measurements) than can be obtained using the coded signals (pseudorange or code measurements). The phase measurements are either processed separately to produce a position measurement which is then used to aided the inertial navigation solution, or are processed directly in with the inertial measurements in a so-called "tightly coupled" architecture (Scherzinger, 2000).

The residual errors left in the differenced phase measurements can be summarized as:

- $\quad$ phase ambiguity (number of cycles)
- $\quad$ ionospheric delays

- tropospheric delays

- receiver noise

The essential requirement to achieving $\mathrm{cm}$ level accuracy is to resolve the cycle ambiguity inherent in each phase measurement to an integer, which is referred to as "ambiguity resolution". Various techniques are used to do ambiguity resolution, the success and reliability of which is primarily dependent upon correcting/reducing the propagation delays as much as possible. The closer the rover is to the reference stations the more the delays cancel out in the differences, while having dual or triple frequency measurements makes the ionospheric delay more readily observable.

1.3.1 Single Reference Station Processing: In this method a single dual or triple frequency GNSS reference receiver is set up close to the project area to be mapped and its location surveyed. The raw phase and code measurements observables from the reference receiver are then transmitted to the roving receiver over a radio link or logged for post-processing. Here the limiting factor becomes the maximum distance the rover can be from the reference stations before the atmospheric delays no longer cancel out and the correct ambiguities can no longer be determined. Depending upon the location (which determines the magnitude of the spatial variations in the ionosphere) this can be no more than about $20 \mathrm{~km}$. This type of distance is fine for small mapping projects such as from land vehicles and unmanned aerial vehicles (UAV's), but is not efficient for typical airborne mapping projects.

1.3.2 Network Reference Station Processing: As its name implies, Network Reference Station processing involves the use of a network of GNSS reference stations instead of a single station. In this scenario, the observables from the network of stations are used to measure the atmospheric delays within the networks and produce a highly accurate model of the local ionosphere. This model and data from the network are then used to generate a set of artificial GNSS observables continuously follows the rover. This Virtual Reference Station or VRS is then processed as if it is a single fixed reference station at the rover location, thus eliminating the baseline separation issue. Such an approach is ideal for $\mathrm{cm}$ level post-processed carrier phase DNSS processing over large areas, and can take advantage of existing Continuously Operation Reference Station (CORS) networks. However in areas where there are no CORS the cost of establishing a dedicated network of receivers can be significant.

\subsection{Precise Point Positioning (PPP)}

Precise Point Positioning (PPP) is a real-time and postprocessed augmentation technique that uses a global network of monitoring stations to precisely estimate and correct the orbit and clock measurements. These precise ephemeris data are then used to reduce the overall error in the code and carrier phase measurements to levels that enables the other error sources to be estimated to decimetre and even centimetre level accuracy, all without the use of local reference stations (Zumberge et al., 1997, Y. Gao..). This has enormous potential for improving the efficiency and lowering the cost of mobile mapping especially in regions where existing reference station networks don't exist or have inadequate coverage to support high accuracy postprocessed DGNSS positioning.

Sources of precise ephemeris include the International GNSS Service (IGS) which provides freely distributed corrections of 
varying accuracy and delay. For the highest level of postprocessed accuracy typically the Rapid or Final products are used which are available 17-41 hours and 12-18 days later respectively (IGS, 2016).

A fundamental limitation for high accuracy PPP is the time it takes to converge to full accuracy. Convergence requires estimation of residual error sources from noise, which is made observable through the satellite change in geometry. Depending upon the constellations used and the signal environment, this can take anywhere from 20 minutes for the horizontal position to anywhere up to 120 minutes for vertical position (Gao et al., 2006). A key advantage of post-processing is the ability to compute both forward and reverse in time solutions which are then combined to eliminate/reduce the periods of convergence. However the duration of the mission must still be longer than convergence time for this to be effective.

\section{TRIMBLE CENTERPOINT RTX}

The Trimble Centerpoint RTX (Real-Time Extended) is an innovative multi-frequency GNSS positioning technology that combines the high accuracy of reference-station based Differential GNSS with the highly productive wide-area coverage of global satellite corrections. First introduced in 2011 supporting GPS and GLONASS (Leandro, 2011), it has now been expanded to include QZSS, BDS and Galileo (Brandl, 2014).

The Trimble approach is unique to most PPP solutions in that it employs its own dedicated precise ephemeris correction service and derives integer level ambiguities for accuracy approaching that of RTK.

The Trimble CenterPoint RTX monitoring network is comprised of approximately 100 high-performance GNSS receivers distributed globally, which track GPS, GLONASS, BDS, QZSS and Galileo.

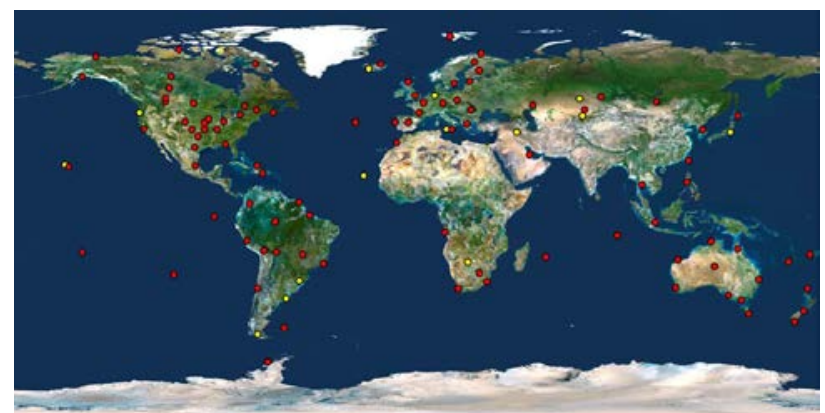

Figure 2: Trimble CenterPoint RTX Tracking Network

The reference stations used in the network are Trimble survey grade receivers, coupled with high performance geodetic antennas that are fully characterized and calibrated.

Data from the stations in the network are transmitted continuously to redundant operation centres located around the globe. The operation centres themselves contain redundant communication and processing servers ensuring seamless, reliable data. The processing servers continuously monitor the health of the reference stations around the world and automatically reconfigure the network when problems are detected. Network processors then generate the precise orbit, clock and observation biases for any location on the Earth at a rate of $1 \mathrm{~Hz}$.
The precise data are then transmitted in real-time using a compressed CMRx message format over satellite and the internet, and logged to a data base for post-processed applications.

\section{POST-PROCESSED RTX AIDED- INERTIAL PROCESSING FOR MOBILE MAPPING}

The POSPac Mobile Mapping Suite (POSPac MMS) is a highly sophisticated GNSS-Aided Inertial post-processing package designed for maximizing the performance of mobile mapping using the Trimble GNSS + inertial products produced by Applanix (a subsidiary of Trimble).

Using the precise data derived from the real-time CenterPoint RTX system, a new high-accuracy post-processed RTX-Aided inertial processing method has been developed for POSPac MMS, enabling robust, $\mathrm{cm}$ level positioning to be achieved for mobile mapping without reference stations.

The Post-processed RTX (PP-RTX) implementation in POSPac is comprised of three components:

1. A web-based service that provides the CenterPoint RTX information along the rover trajectory to be post-processed.

2. A QC step that processes the information from the service with the raw rover observables in forward and reverse time to generate the convergence-free PPRTX GNSS solution

3. Generation of the final RTX-Aided Inertial navigation solution using a Kalman filter and optimal smoother processing

\subsection{Post-processed CenterPoint RTX Service}

The post-processed CenterPoint RTX service is accessed automatically from the POSPac MMS via an internet connection. A compressed trajectory derived from the real-time solution is uploaded to the RTX server infrastructure and used to retrieve a set of data comprised of orbit, clock and additional local bias information derived from the real-time CenterPoint RTX service. Unlike a traditional PPP solution that uses IGS or equivalent ephemeris data that can take several days to compute, this information is available within 1 hour after the mission.

\subsection{Post-processed RTX-Aided Inertial Solution}

The PP-RTX solution is computed using dual frequency pseudorange and carrier phase measurements from the GPS, GLONASS, QZSS and BeiDou satellites.

The simplified equation for the carrier phase and pseudorange measurements from a receiver to a given satellite can be written as follows (Leandro et al, 2011, Doucet et al, 2012):

$$
\begin{aligned}
\Phi_{i}= & \rho+c(d T-d t)+T-I_{i}+A_{i}-a_{i}+\frac{W_{\Phi}-w_{\Phi}}{\lambda_{i}}+B_{\Phi, i} \\
& -b_{\Phi, i}+\lambda_{i} N_{i}+m_{\Phi, i}+\epsilon_{\Phi, i} \\
\mathrm{P}_{i}= & \rho+c(d T-d t)+T+I_{i}+A_{i}-a_{i}+B_{\mathrm{P}, i}-b_{\mathrm{p}, i}+ \\
& m_{p, i}+\epsilon_{\mathrm{p}, i}
\end{aligned}
$$

where: 
$\Phi_{i} \quad$ Carrier-phase measurement for frequency i

$\mathrm{P}_{i} \quad$ Pseudorange measurement for frequency i

$\rho \quad$ Receiver antenna to satellite range

c Speed of light

$d T \quad$ Receiver clock error

$d t \quad$ Satellite clock error

$T \quad$ Tropospheric delay

$I_{i} \quad$ Ionospheric delay for frequency i

$A_{i} \quad$ Receiver ant offset and variation for frequency i

$a_{i} \quad$ Satellite ant offset and variation for frequency i

$W_{\Phi} \quad$ Receiver antenna phase wind-up

$w_{\Phi} \quad$ Satellite antenna phase wind-up

$B_{\Phi, i} \quad$ Carrier phase receiver bias for frequency i

$b_{\Phi, i} \quad$ Carrier phase satellite bias for frequency i

$B_{\mathrm{P}, i} \quad$ Pseudorange receiver bias for frequency i

$b_{\mathrm{P}, i} \quad$ Pseudorange satellite bias for frequency i

$\lambda_{i} \quad$ Carrier wavelength for frequency i

$N_{i} \quad$ Integer ambiguity for frequency i

$m_{\Phi, i} \quad$ Carrier phase multipath for frequency i

$m_{\mathrm{P} i} \quad$ Pseudorange multipath for frequency i

$\epsilon_{\Phi, i} \quad$ Carrier measurement error and residual errors

$\epsilon_{\mathrm{P} i} \quad$ Pseudorange measurement error and residual errors

Through measurement, modelling and estimation, the PP-RTX solution computes and applies corrections to the carrier phase measurements to an accuracy of a few cm's, allowing the integer ambiguities to be resolved and a high accuracy position solution to be produced.

The precise satellite clocks and orbits received from the RTX server are used to eliminate the contributions of the satellite clock error and satellite position error in the computation of the receiver position. Additional global and/or regional Ionospheric corrections are also computed, which are then applied to help reduce the time of convergence.

High accuracy models are used in both the computation of the satellite clocks and orbits and by the positioning engine to further reduce the error in the phase measurements:

- Satellite and receiver antenna corrections

- $\quad$ Solid Earth tides

- $\quad$ Pole tides

- Ocean loading effects

- Relativistic corrections

- $\quad$ Phase wind up

- $\quad$ Satellite code and carrier phase biases

The error components in the measurements after applying the precise clock, orbits and models are dominated by the ionospheric and tropospheric delays, receiver clock errors, receiver biases, multipath and of course the phase ambiguities themselves.

The PP-RTX positioning engine uses a Kalman filter to estimate the ambiguities, construct the position, and estimate and attenuate the remaining errors.

The receiver clock error is observable and estimated as part of the engine. The first order error due to ionospheric delay is mitigated by constructing combinations of the measurements between the different frequencies to produce the so-called ionospheric-free measurements (Leandro et al, 2011).
The geometry-free ionosphere-free linear combination, i.e. wide-lane carrier-phase minus narrow-lane code (Garbor and Nerem, 2002), provides direct access to the wide-lane ambiguities. However it still contains the receiver and satellite biases. The satellite phase biases are obtained from the RTX network processor system (Leandro et al, 2011). The remaining biases are obtained by combining the divergence-free phase combination and the code measurement (Leandro et al, 2011). The ionospheric phase combination and ionospheric code combination are employed to estimate the ionosphere delay to each satellite. At this point the ionosphere delays, the wide-lane ambiguities and the phase biases are known and/or derived, and only the modelling of the tropospheric delay remains. The total tropospheric delay can be represented by

$$
T=m_{h}(\epsilon) T_{h}^{Z}+m_{w}(\epsilon) T_{w}^{Z}
$$

where $T$ is the total slant delay at an elevation angle $\epsilon, T_{h}^{Z}$ and $T_{w}^{z}$ are the hydrostatic and wet zenith delays respectively, and $m_{h}(\epsilon)$ and $m_{w}(\epsilon)$ are corresponding mapping functions. Troposphere delay prediction models such as Hopfield, Saastamoinen, and GTP2 $\mathrm{w}$ provide accuracies of up to a few decimetres in the zenith direction. The residual error is caused by the variability of the temperature, pressure, and water vapour present at the time of observation, and is modelled and estimated in the Kalman filter. Optimization of the error model using empirical data sets was critical for achieving high performance for airborne kinematic surveys due to the potential for rapidly changing altitude of the rover.

The time it takes the PP-RTX engine to converge to the final correct ambiguities is a function of the multipath environment and the geometry of the satellites which make the other errors observable. This can range from less than 1 minute to over 15 minutes depending upon the availability of a local Ionospheric model and the quality of observables (Nardo et al, 2015). However since the PP-RTX is running both in the forward and reverse direction with the final output being the combination of the two, as long as the mission itself is more than about 45 minutes with no loss of satellite lock (such as in aircraft applications), the final solution is "convergence free".

The highly accurate PP-RTX solution is processed along with the code and Doppler measurements from GPS and GLONASS in the Aided-Inertial Kalman filter to produce a cm-level, highly robust, smoothed position and orientation solution (SBET) at $200 \mathrm{~Hz}$.

\section{AIRBORNE ACCURACY ASSESSMENT}

This section presents some typical results of the PP-RTX aided inertial technology when used for airborne mapping applications.

\subsection{Methodology}

The POSPac PP-RTX aided inertial positioning solution was assessed against a reference trajectory for 100 airborne datasets (equivalent to 220 hours of data) collected by Applanix POS AV customer in various countries around the world (e.g. Canada, USA, South Africa, UK, Norway, Australia, New Zealand, etc.) from 2011 to 2015. The data sets cover both fixed wing and helicopter platforms, and different altitudes ranging from $160 \mathrm{~m}$ to $8758 \mathrm{~m}$ with an average height above ground of $1392 \mathrm{~m}$. The reference trajectories were generated using POSPac and the Applanix Smartbase ${ }^{\mathrm{TM}}$ (ASB) module, which is 
Applanix's aided inertial VRS post-processing technology. All reference trajectories were inside their respective reference station networks to ensure that the estimated positional accuracy was 1-2 cm RMS in the horizontal and 2-4 cm RMS in the vertical throughout most of the trajectories. Figure 3 shows an example of a typical airborne survey trajectory and its corresponding ASB network. The reference trajectories and POSPac PP-RTX solutions were computed at $200 \mathrm{~Hz}$ and down sampled to $10 \mathrm{~Hz}$ for plotting and analysis. The position differences between the PP-RTX and ASB solutions were then computed and analysed.

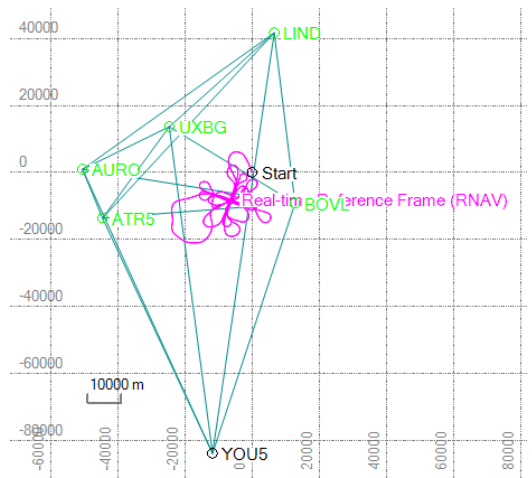

Figure 3: Airborne Survey Trajectory and ASB ${ }^{\mathrm{TM}}$ Network

\subsection{Results}

Figure 4 shows the POSPac PP-RTX and ASB processing modes of all 100 dataset. Each colour in the plot represents a dataset, and the majority of the epochs are of fixed integer solution. Figure 5 shows the North, East and Down position differences between the PP-RTX and ASB solutions, while Table 1 presents the associated statistics of the differences.

The RMS of the differences between the two solutions in the North, East and Down components are $0.021 \mathrm{~m}, 0.020 \mathrm{~m}$ and $0.056 \mathrm{~m}$ respectively. Such close agreement indicates that the POSPac PP-RTX solution is virtually equivalent to the POSPac ASB solution, and hence is sufficient to meet the requirements for a majority of airborne mapping applications.
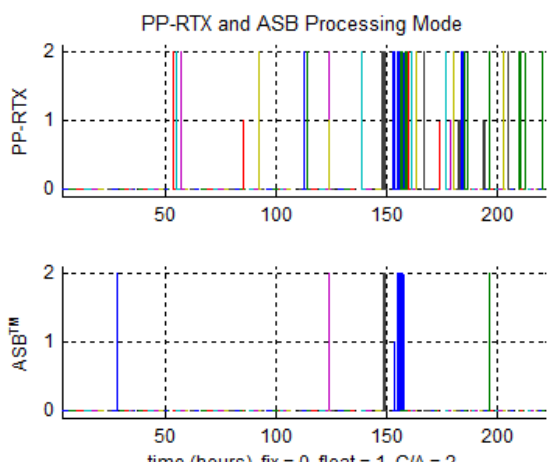

Figure 4: Processing Mode, PP-RTX and ASB ${ }^{\mathrm{TM}}$
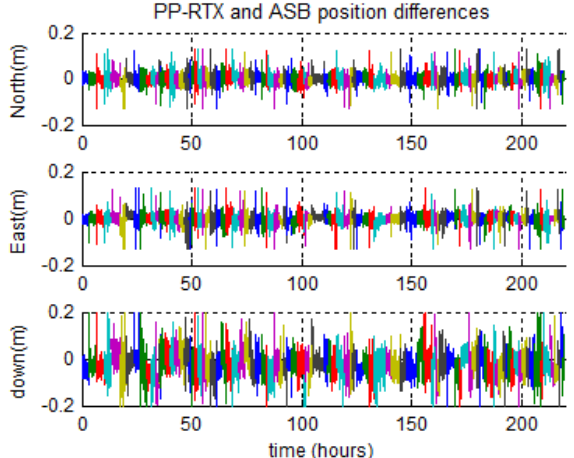

Figure 5: Position Difference, PP-RTX vs. ASB

\begin{tabular}{|c|c|c|c|c|c|c|}
\hline $\begin{array}{c}\text { Position } \\
\text { components }\end{array}$ & $\begin{array}{c}\text { RMS } \\
\text { (m) }\end{array}$ & $\begin{array}{c}\text { Sigma } \\
\text { (m) }\end{array}$ & $\begin{array}{c}\mathbf{6 8 \%} \\
\text { (m) }\end{array}$ & $\begin{array}{c}\mathbf{9 5 \%} \\
\text { (m) }\end{array}$ & $\begin{array}{c}\mathbf{9 9 \%} \\
(\mathbf{m})\end{array}$ & $\begin{array}{c}\mathbf{9 9 . 9 \%} \\
(\mathbf{m})\end{array}$ \\
\hline North (m) & 0.021 & 0.021 & 0.014 & 0.034 & 0.066 & 0.135 \\
\hline East (m) & 0.020 & 0.020 & 0.012 & 0.032 & 0.066 & 0.155 \\
\hline Down (m) & 0.056 & 0.050 & 0.052 & 0.110 & 0.156 & 0.245 \\
\hline
\end{tabular}

Table 1: Position Difference Statistics, PP-RTX vs. ASB

One can notice that some spikes greater than $10 \mathrm{~cm}$ appear in the difference plots, which are also reflected in the $99.9 \%$ statistic numbers. These spikes are mainly caused by the GNSS multipath at the rover or short outages in the observables where a fixed integer solution cannot be maintained in the PP-RTX and/or ASB modes. This usually occurred only at the beginning or end of a mission when the aircraft might have been parked close to a hanger. This is illustrated in Figure 6 which shows the difference plots from one dataset.

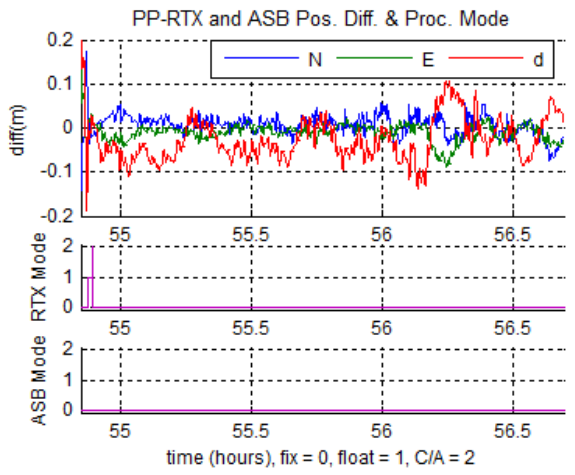

Figure 6: Position Difference, PP-RTX vs. ASB for one dataset

\subsection{Convergence Analysis}

4.3.1 Initial Convergence Times, Forward and Reverse: To analyse the forward and reverse convergence times, 41 out of the 100 datasets were chosen that contained "good" GNSS observables during the first and last hour of their missions. A "good" dataset is characterized by gap-free data, minimal cycle slips, high signal-to-noise ratio signals, and low DOP values. Using only the "good" data sets for this analysis gives a clearer picture of how quickly and reliably the PP-RTX technology can estimate the residual biases free from other factors that can delay the final convergence (such as high DOP or cycle slips which can happen during take-off and landing).

For each data set the forward and backward PP-RTX position solutions were differenced with the ASB reference solution to produce the 3D radial differences at each epoch. Figures 7 and 8 show the 3D differences and the processing mode for the 
forward and reverse passes for all 41 dataset. Each colour in the plot represents a separate dataset. The black line in the top plots shows the average of the 3D radial difference, while the one in the bottom plots represents the average processing mode. On average it takes 11.5 and 20.4 minutes to converge to $0.150 \mathrm{~m}$ and $0.075 \mathrm{~m}$ 3D RMS in the forward pass, while in the backward pass it takes 11.0 and 18.0 minutes to reach the same 3D RMS values.
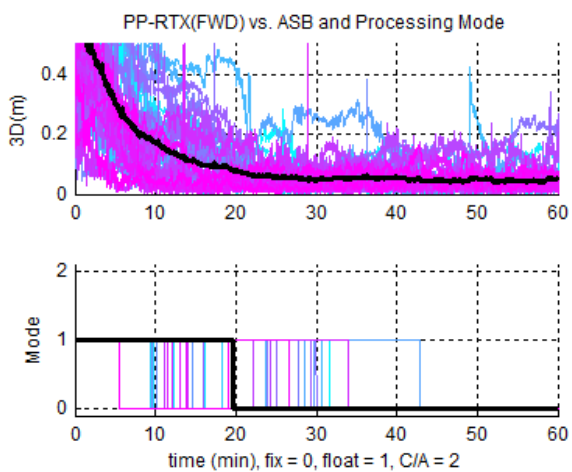

Figure 7: Forward PP-RTX vs. ASB and Processing Mode
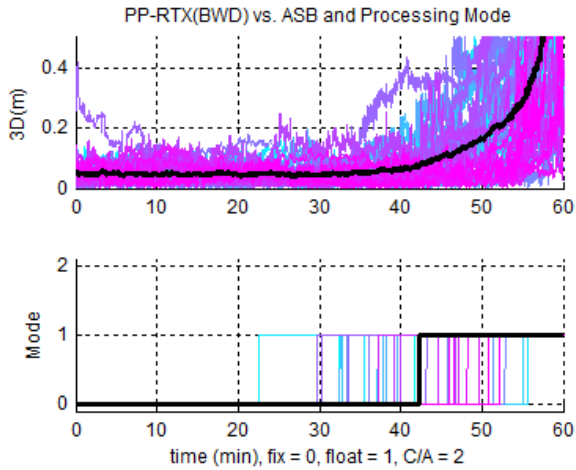

Figure 8: Backward PP-RTX vs. ASB and Processing Mode

From the mode plots it can be seen that the average time for the processing mode to indicate the equivalent of fixed ambiguities in the forward and backward passes are 19.7 and 17.7 minutes respectively, which is essentially the same time it takes on average to converge to $0.075 \mathrm{~m}$ 3D RMS (about 20 minutes). Hence for the purpose of this analysis, convergence is defined as either the processing mode indicating a fixed solution, or when the $3 \mathrm{D}$ position error is equal to $0.075 \mathrm{~m}$ or less.

The minimum and maximum times to fix the ambiguities in the forward and reverse directions (figures 7 and 8) are 5.4 and 42.9 4.4 and 37.5 minutes respectively. The convergence time and subsequent time to fix the ambiguities depends mostly on the accuracy of the regional ionospheric corrections the RTX server provides to the PP-RTX GNSS processor. This is illustrated in Figures 9 and 10, which show the $1-\sigma$ accuracy of the ionospheric corrections for the measurements to each satellite vehicle number (SNV) for the datasets with the fastest (5.4 min) and slowest (42.9 $\mathrm{min})$ time to fix ambiguities. The standard deviations of the corrections in Figure 9 are significantly smaller than in Figure 10, thus ensuring faster convergence and ambiguity resolution.

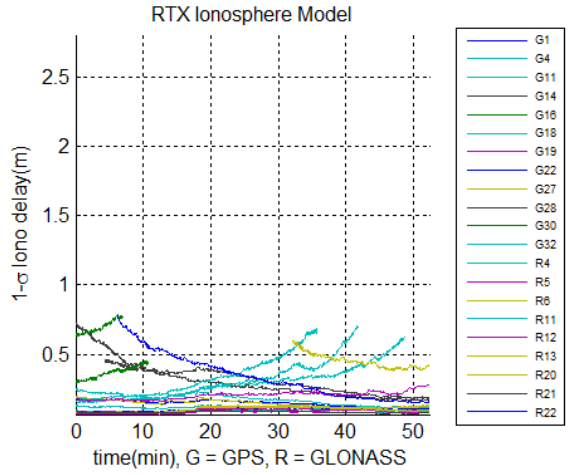

Figure 9: Ionospheric correction accuracy (1- $\sigma)$ for the dataset with the fastest position fixing (5.4 min)

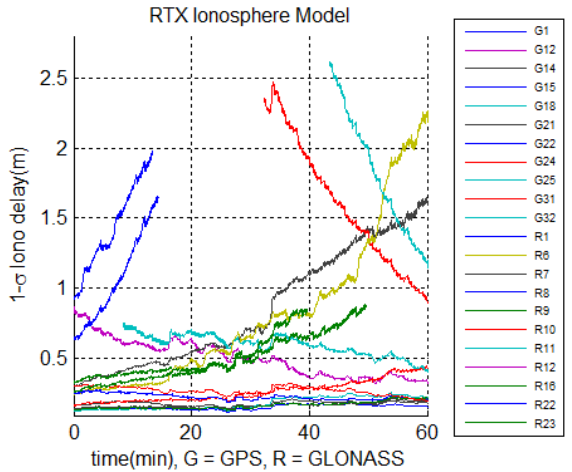

Figure 10: Ionospheric correction accuracy (1- $\sigma)$ for the dataset with the slowest position fixing (42.9 min)

In post-processing the forward and backward PP-RTX solutions are combined to provide a fixed processing mode and centimetre level positioning accuracy for the entire trajectory, as illustrated in Figure 11.
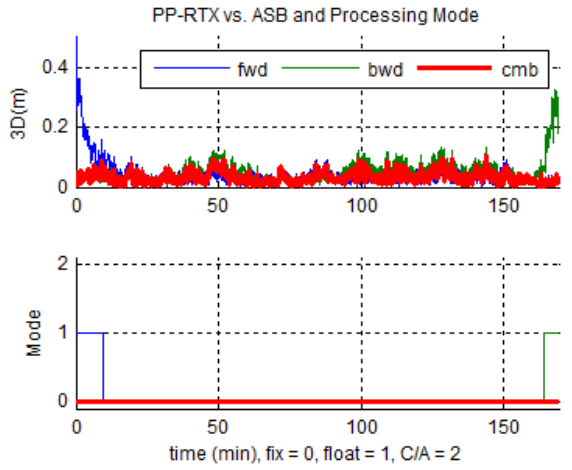

Figure 11: Forward, Backward \& Combined PP-RTX vs.

$\mathrm{ASB}^{\mathrm{TM}}$ and Processing Mode (Typical Case)

Hence if the trajectory itself is longer than the time to convergence, PP-RTX can produce a combined centimetre level convergence-free solution for the entire trajectory. For the 41 "good" data sets analysed the maximum time to convergence was about 42.9 minutes, while the average time to convergence was 20 minutes. This implies that if extra care is taken on how the data are collected, airborne trajectories as short as 45 minutes can produce the highest accuracy results, without any risk of non-convergence.

When considering at all 100 airborne data sets, which included missions where the DOP could be poor at the beginning and end of the mission or cycle slips might have occurred at take-off or 
landing, the longest time to reach convergence was 72.48 minutes in the backwards direction, or almost the entire trajectory (Figure 12). However in this case the forward solution took just over 20 minutes to converge, which meant the combined solution for the entire flight was still convergence free.
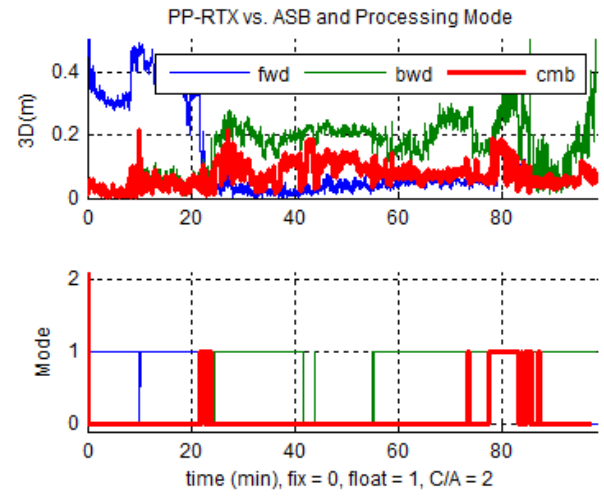

Figure 12: Forward, Backward \& Combined PP-RTX vs. ASB and Processing Mode (Longest Convergence Case)

4.3.2 Re-convergence and Robustness: As with carrier phase post-processed DGNSS-Aided Inertial processing, the accuracy that can be achieved using PP-RTX-Aided Inertial is directly related to the quality of the raw GNSS observables that are collected during the mission. Outages in the GNSS observables are bridged automatically with the inertial solution, and the GNSS ambiguities are re-computed in the forward and reverse direction to produce the combined solution. Unlike DGNSS which can re-resolve ambiguities within seconds after outages, PP-RTX needs enough clean data before and after the outage to initially converge to the final accuracy. However once it has converged, the PP-RTX solution can re-converge quite quickly if the signals are interrupted since the filter retains memory of the converged ionosphere and troposphere delay estimates.

The robustness and speed of re-convergence is illustrated in Figure 13. In this data set a short outage of the phase measurements for 2 seconds resulted in the PP-RTX solution jumping to C/A code accuracy and then re-converging to integer accuracy. As can be seen in the plot, both the forward and reverse solutions re-converge quite quickly and the combined solution only shows a slight degradation in accuracy during the actual outage itself.
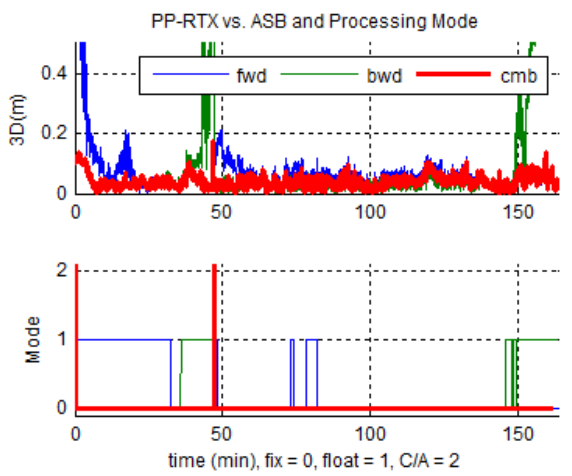

Figure 13: Forward, Backward \& Combined PP-RTX vs. ASB and Processing Mode (with short outages)

\subsection{Limitations}

The results summarized above were obtained for manned aircraft mapping applications which by their nature have a fully open sky, minimal multipath, long duration missions (i.e. at least 1 hour) and very clean GNSS observables without gaps and with minimal cycle slips. Hence PP-RTX is ideally suited for this application. While it can also be used for unmanned aerial mapping applications, it is often much more challenging to get clean observables from these platforms, and the duration of the trajectories tend to be less than about 45 minutes, which makes it difficult to guarantee convergence.

Another application that is well suited for PP-RTX is marine seafloor mapping. Here, much like the airborne application, trajectories tend to be long in duration and the open sky ensures good clean observables.

While some land based mobile mapping applications might also be able to take advantage of PP-RTX in open sky areas, the constant cycle slips and outages experienced in most urban areas make it difficult for PP-RTX to converge.

\section{CONCLUSIONS}

PP-RTX aided-inertial processing is a new GNSS aided-Inertial technology that provides centimetre level post-processed positioning accuracies without the use of local reference stations. This new method integrates the Trimble CenterPoint RTX technology into the Applanix POSPac MMS GNSS-Aided Inertial software for mobile mapping, where it has been optimized for airborne surveys by catering to the larger variations in the troposphere delay, and by processing raw code and Doppler measurements in cases of partial GNSS outages.

The POSPac MMS PP-RTX aided-inertial technology:

- was shown to match the North, East and Down position computed using the ASB network Carrier Phase DGNSS processing for 100 airborne data sets to an accuracy of $0.021 \mathrm{~m}, 0.020 \mathrm{~m}$ and $0.056 \mathrm{~m}$ RMS respectively, which is sufficient to meet the accuracy requirements for a majority of mapping applications

- does not need local reference stations

- can obtain carrier phase ambiguity fixing in as little as 5.4 minutes and on average 20 minutes when clean GNSS observables are collected

- $\quad$ provides a convergence-free solution by combining the forward and backward solutions

- $\quad$ is available 1 hour after data collection

\section{FUTURE WORK}

Future work will focus on using more accurate regional ionospheric corrections provided by the RTX servers to reduce convergence time. This will enable POSPac PP-RTX to be used more effectively on shorter trajectories such as those often encountered with Unmanned Aerial Vehicles, and improve robustness for land based mobile mapping applications.

\section{ACKNOWLEDGEMENTS}

The authors would like to thank Joachim Tappe, Ian Murray, Terence Fu, Danut Tabacaru, Catalin Floroiu, Bertram Blake, James Li and James Lutes for their help on the PP-RTX POSPac MMS development, integration, data processing and testing. 


\section{REFERENCES}

Brandl M., M. Brandl, X. Chen, R. Drescher, M. Glocker, H. Landau, A. Nardo, M. Nitschke, D. Salazar, S. Seeger, U. Weinbach, F. Zhang, 2014. Advancing Trimble RTX Technology by adding BeiDou and Galileo. Trimble TerraSat GmbH, Germany.

Doucet K., Herwig M., Kipka A., Kreikenbohm P., Landau H., Leandro R., Moessmer M., Pagels C,. 2012. Introducing Ambiguity Resolution in Web-hosted Global Multi-GNSS Precise Positioning with Trimble RTX-PP, Proceedings of the 25th International Technical Meeting of The Satellite Division of the Institute of Navigation (ION GNSS 2012), Nashville, Tennessee, pp. 1115-1125

Gao, Y., Zhang, Y., Chen, K., "Development of a Real-Time Single-Frequency Precise Point Positioning System and Road Test Results," Proceedings of the 19th International Technical Meeting of the Satellite Division of The Institute of Navigation (ION GNSS 2006), Fort Worth, TX, September 2006, pp. 22972303.

Garbor M.J., Nerem R.S, 2002. Satellite-Satellite SingleDifference Phase Bias Calibration as Applied to Ambiguity Resolution. Journal of the Institute of Navigation, Vol. 49, No. 4, pp. 223-242.

Hutton J., Mostafa M.M.R, 2005. 10 Years of Direct Georeferencing for Airborne Photogrammetry, Photogrammetric Week 2005, Stuttgart, Germany.

Hutton J., Ip A., Bourke T., Scherzinger B., Gopaul N., Canter P., Oveland I., Blankenberg L., 2008. Tight Integration of GNSS Post-processed Virtual Reference Station with Inertial Data for Increased Accuracy and Productivity of Airborne Mapping. ISPRS Congress 2008, Beijing, ICWG V/I.

IGS, 2016. IGS products https://igscb.jpl.nasa.gov/components/ prods.html, accessed on 04 April 2016.

Leandro R., et al, 2011. RTX positioning: The Next Generation of cm-accurate Real-time GNSS Positioning. Trimble TerraSat $\mathrm{GmbH}$, Germany. Proceedings of the 24th International Technical Meeting of The Satellite Division of the Institute of Navigation (ION GNSS 2011), Portland, Oregon, pp. 1460 1475.

Mostafa M.M.R, Hutton J., 2001. Direct Positioning and Orientation Systems, How Do they Work? What is the Attainable Accuracy? Proceedings ASPRS Annual Meeting 2001, St. Louis, MO USA.

Scherzinger Bruno M., 2000. Precise Robust Positioning with Inertial/GPS RTK, Proceedings of the 13th International Technical Meeting of the Satellite Division of The Institute of Navigation (ION GPS 2000), Salt Lake City, UT, September 2000, pp. 155-162

Zumberge J.F., Heflin M.B., Jefferson D.C., Watckins M.M. 1997. Precise point positioning for the efficient and robust analysis of GPS data from large networks. Journal of Geophysical Research, Vol. 102, No. B3, pp. 5005-5017. 